\title{
Modelos matemáticos para el análisis de la selectividad antileishmanial de derivados tiadiazínicos.
}

\section{Mathematical model for the analysis of antileishmanial selectivity of thiadiazin derivatives.}

Lianet Monzote Fidalgo Universidad Técnica de Machala (Machala - Ecuador)

Julieta Coro Bermello Instituto de Medicina Tropical Pedro Kourí (Cuba) monzote@ipk.sld.cu
Taymara Hernández Ortega Universidad de las Ciencias Informáticas

(Cuba)

Revista Cumbres Vol.4 №1

Versión impresa ISSN 1390-9541

Versión electrónica ISSN 1390-3365

http://investigacion.utmachala.edu.ec/revistas/index.php/Cumbres 


\title{
RESUMEN
}

En la literatura se ha reportado la actividad de derivados de tetrahidro-2H-1,3,5-tiadiazín-2-tionas (THTT) frente a amastigotes intracelulares de Leishmania donovani. Sin embargo, a pesar de que en estos ensayos se han obtenido valores bajos de Concentración Inhibitoria Media (CI50) frente a amastigotes intracelulares, los índices de Concentración Citotóxica Media en células mamíferas (CC50) resultan también bajos, evidenciándose actividad celular inespecífica. El objetivo de este trabajo es obtener modelos matemáticos mediante el empleo de descriptores moleculares cuánticos y físico-químico, con el fin de analizar la relación de estos índices con la actividad y citotoxicidad de derivados bis-tiadiazínicos como fármacos potenciales frente a L. donovani. Fueron utilizados descriptores cuánticos y físico-químicos para la creación de modelos predictivos de relación cuantitativa estructura-actividad y estructura-citotoxicidad de 18 compuestos derivados de la THTT con actividad in vitro frente a L. donovani. Las geometrías moleculares de los compuestos fueron optimizadas a través del hamiltoniano semiempírico AM1. Los modelos fueron obtenidos utilizando Regresión Lineal Múltiple y validados mediante la técnica de validación cruzada (dejando uno fuera). Se obtuvieron modelos con valores aceptables de los parámetros estadísticos observados para la variable CC50 ( $\mathrm{R} 2=0,80 ; \mathrm{Q} 2=0,80)$, no siendo así para la variable IC50 ( $\mathrm{R} 2=0,47 ; \mathrm{Q} 2=0,80)$. Los modelos obtenidos evidencian que el Calor de Formación influye de manera significativa en ambas propiedades, lo cual podría explicar la actividad inespecífica de estos compuestos. Lo anterior, unido al manejo del resto de las variables con influencia significativa encontradas en el modelo obtenido para CC50 puede contribuir al diseño de nuevos derivados THTT con actividad específica frente a L. donovani.

Palabras clave: Tetrahidro-2H-1,3,5-tiadiazín-2-tionas, Leishmania donovani, modelos matemáticos, descriptores quánticos y fisicoquímicos

\begin{abstract}
Literature has reported activity of tetrahydro- $2 \mathrm{H}-1,3,5$-thiadiazin-2-thiones (THTT) derivative against intracellular amastigotes of Leishmania donovani. However, although lower values were obtained of Mediam Inhibitory Concentration (IC50) against intracellular amastigotes, in these studies the Mediam Cytotoxic Concentration (CC50) rates evaluated in mammalian cells are also low, showing unspecific cellular activity. The objective of this work was to obtain mathematical models through quantum and physicochemical descriptors, with the aim to analyzing the relation between the index with the activity and cytotoxicity of THTT as potential drugs against L. donovani. Quantum and physicochemical descriptors were used for the creation of predictive modeling with quantitative structure-activity and structure-cytotoxicity of 18 compounds derived from THTT with in vitro activity against L. donovani. The molecular geometries of the compounds were optimized through the
\end{abstract}

\section{$2 \quad$ Cumbres}


AM1 semiempirical Hamiltonian. The models were obtained using Multiple Linear Regression and validated by cross-validation technique (leave one out). Models with acceptable values of the observed statistical parameters were obtained for CC50 ( R2 = 0.80; Q2 = 0.80). Unfortunately the behavior not was the same for IC50 $(\mathrm{R} 2=0.47 ; \mathrm{Q} 2=0.80)$. The models obtained show that the Heat of Formation has a significant influence on both properties (IC50 and CC50), which could explain the nonspecific activity of these compounds. This result joined to the handling of other variables with significant influence found in the model obtained for CC50, can help in the design of new THTT derivatives with specific activity against L. donovani.

Keywords: Tetrahydro- 2H-1,3,5-thiadiazin-2-thiones, Leishmania donovani, mathematical models, quantum and physicochemical descriptors.

\section{INTRODUCCIÓN}

Leishmaniasis es el término que se asigna a las distintas manifestaciones clínicas causadas por alrededor de 16 especies parásitas del género Leishmania. Estas pueden ser clasificadas en: cutáneas, mucocutáneas y viscerales. Esta última es la forma aguda de la enfermedad, causada por especies del complejo L. donovani (Alvar et al., 2012).

Los fármacos disponibles para este padecimiento son escasos y presentan varias restricciones para su empleo. Entre los inconvenientes de estas terapias se incluyen: alta toxicidad, vía de administración parenteral, respuesta variable al medicamento, precios prohibitivos y resistencia del parásito (Monge-Maillo y López-Vélez 2013, Nagle et al., 2014).

En la literatura se ha reportado la actividad de derivados de tetrahidro-2H-1,3,5-tiadiazín-2-tionas (THTT) frente a varias especies de Leishmania (Monzote et al., 2004, Coro et al., 2005, Coro et al., 2006, Coro et al., 2008, Coro et al., 2011). Los compuestos ensayados poseen uno o dos anillos tiadiazínicos en su estructura (Figura 1). En particular para las bis-THTT, se han obtenido valores bajos de Concentración Inhibitoria Media (CI50) en estas evaluaciones, incluso comparables con las terapias actuales, sin embargo, valores de Concentración Citotóxica Media (CC50) fueron también bajos (Coro et al., 2005). En la Tabla 1 se muestran los valores experimentales asociados a cada uno de los compuestos moleculares.

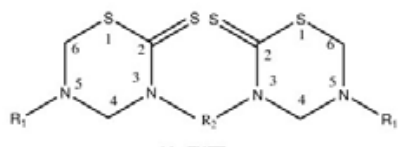

Figura 1: Núcleo estructural base bis-THTT. En la tabla 1 se describen las estructuras químicas obtenidas para R1 y R2. 
Tabla 1: Estructura química de las bis-THTT y valores experimentales de actividad antileishmanial (CI50) y citotoxicidad (CC50) utilizados como base de datos para la creación de los modelos.

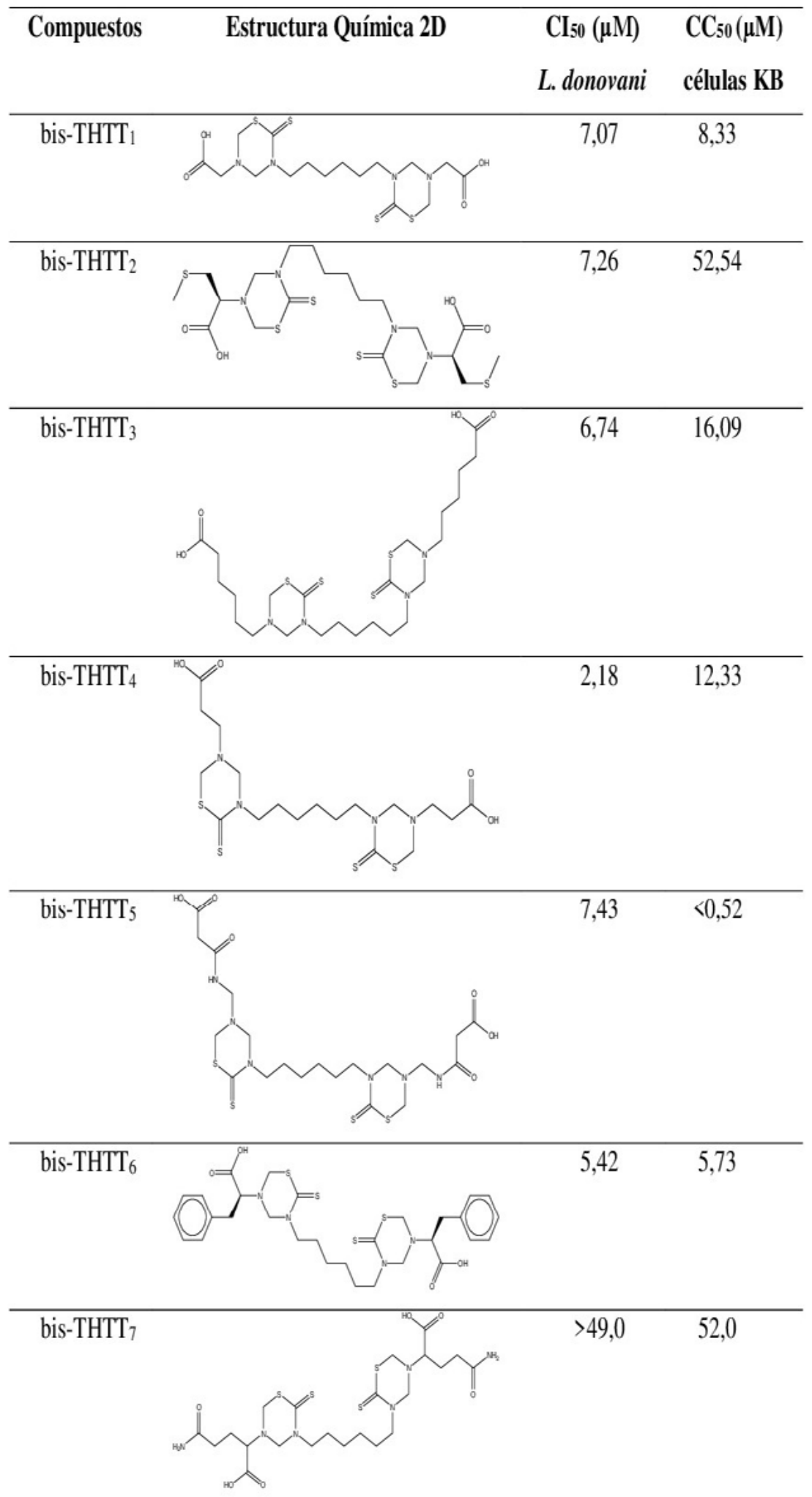




\section{PRE-PRINT}

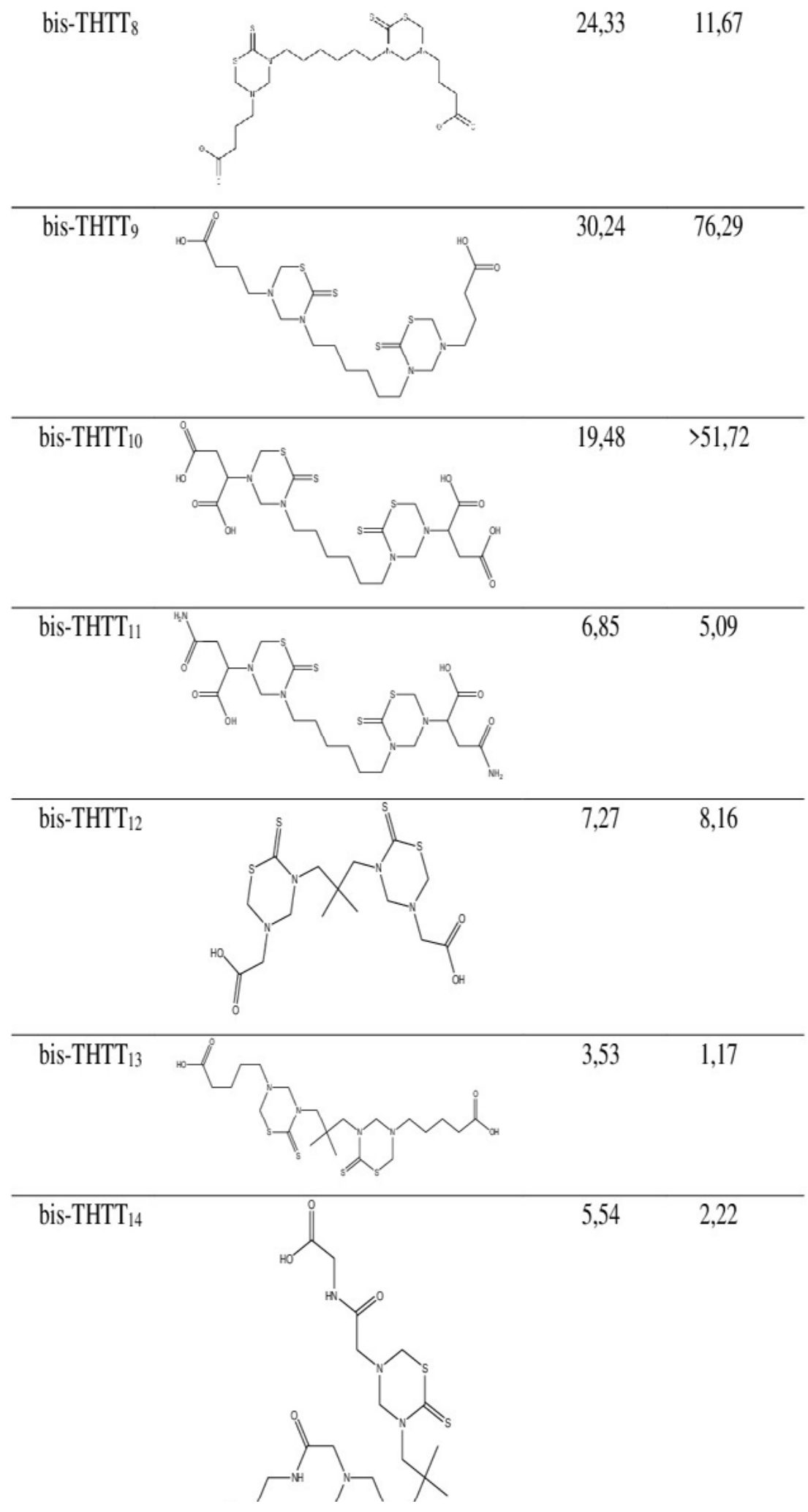




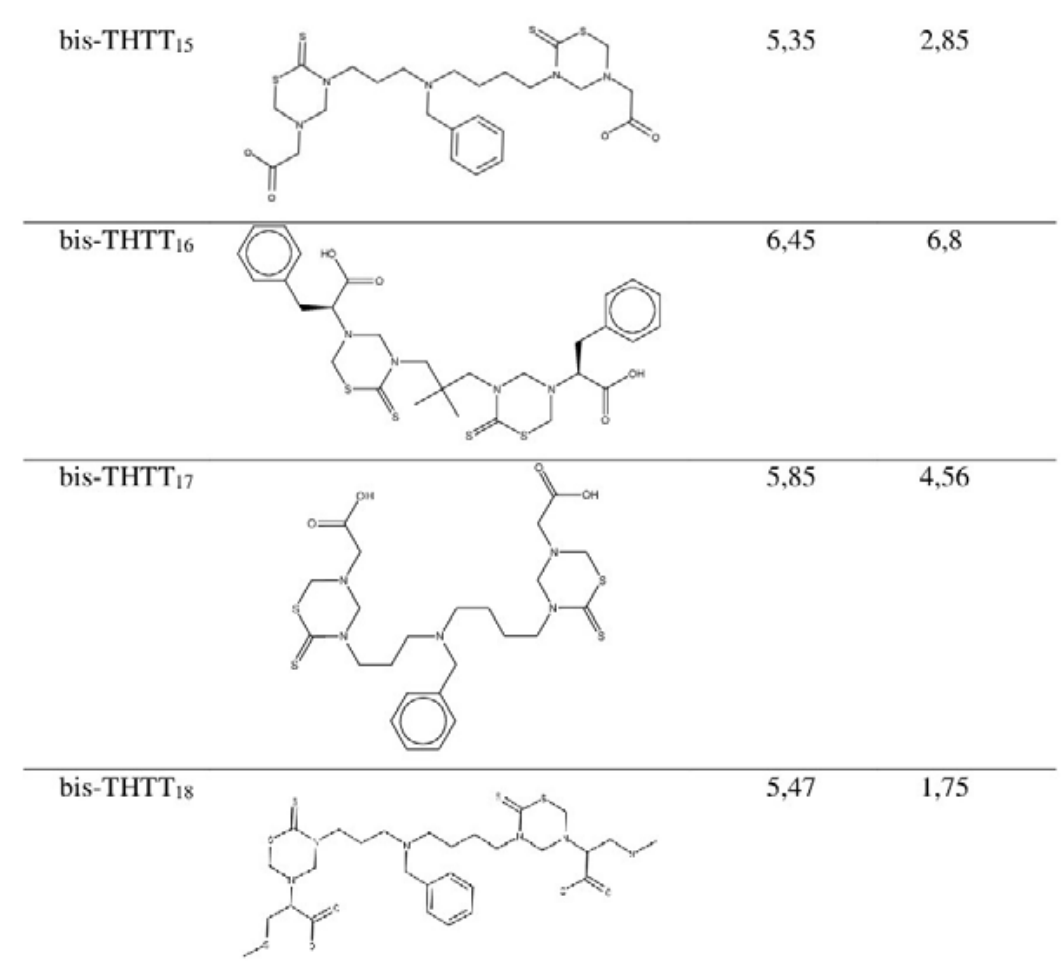

CI50: Concentración que causa un 50\% de inhibición del crecimiento de amastigotes intracelulares de L. donovani.

CC50: Concentración citotóxica media que causa un 50\% de inhibición delö creciemiento de células KB.

El objetivo de este trabajo es, obtener modelos matemáticos mediante el empleo de descriptores moleculares cuánticos y físico-químico, con el fin de analizar la relación de estos índices con la actividad y citotoxicidad de derivados bis-THTT como fármacos potenciales frente a L. donovani. El uso de estos modelos podría contribuir a explicar los bajos índices de selectividad frente a esta especie y apoyar al futuro diseño de nuevos derivados bis-THTT con un índice de selectividad apropiado para el desarrollo de fármacos antileishmanial.

\section{MATERIALES Y MÉTODOS}

Las hojas de Moringa oleífera se recolectaron de la unidad académica de Datos: La muestra utilizada en este estudio está conformada por 18 bis-tiadiazinas (Tabla 1), de las cuales se han reportado ensayos in vitro frente a amastigotes intracelulares de L. donovani, utilizando células KB para evaluar la citotoxicidad (Coro et al., 2005, Coro et al., 2006, Coro et al., 2008). 
Selección de los descriptores moleculares: Un total de once descriptores relacionados con las propiedades electrónicas, estéricas e hidrofóbicas fueron determinados y sometidos a un análisis de correlación de variables a través de la matriz de correlación de Pearson. Como resultado de este proceso fueron seleccionados la energía del orbital desocupado de más baja energía (LUMO), energía del orbital ocupado de más alta energía (HOMO), calor de formación (C.F), momento dipolar ( $\mu$ ), energía total de cada sistema molecular (E.T) y las cargas asociadas a los átomos de nitrógeno en los anillos en la posición 3 y 5 (q3 y q5, respectivamente).

En la optimización de las geometrías moleculares y obtención de los descriptores mecano-cuánticos fue utilizado el hamiltoniano semiempírico AM1, implementado en la versión 9.0 del programa MOPAC (James 2008). Se decidió utilizar para la selección de las variables, el algoritmo selección hacia atrás-selección hacia adelante implementado en el paquete Rcmdr-CRAN incorporado a la versión 3.11 de R (Lumley 2001).

Obtención de los modelos matemáticos: Para el tratamiento de valores omitidos se seleccionó la imputación por media aritmética. En el caso de la existencia de rangos se decidió dejar el valor límite reportado.

La Regresión Lineal Múltiple (RLM) fue utilizada en la construcción de los modelos matemáticos implementado en el paquete Rcmdr-CRAN también incluido en el programa R 3.1.1. Para su diagnóstico se tuvieron en cuenta los valores del coeficiente de correlación ajustado (R2), el estadístico de Fisher (F) y el valor de probabilidad (p). Debido al tamaño de la muestra, solo fue aplicado el método de validación interna Validación Cruzada con k-iteraciones en los modelos finales seleccionados. Los grupos fueron creados dejando solo un caso fuera (método comúnmente abordado como leave one out). Este análisis se realizó a través de la métrica Q2 calculado en este trabajo haciendo uso del paquete cvq2 implementado en la versión 3.1.1 del programa $\mathrm{R}$ (Cramer et al., 1988).

En el estudio de los valores atípicos, se tuvo en cuenta el resultado arrojado por el test de valores atípicos de Bonferroni obtenidos usando Rcmdr-CRAN. Esto permitió analizar posibles valores influyentes, cuya presencia en la base datos pudiera afectar los parámetros de validación del modelo, valores y grado de significación de los coeficientes atribuidos a cada variable.

\section{RESULTADOS Y DISCUSIÓN}

Modelo para el análisis de la citotoxicidad: Modelos con una, dos y tres variables fueron obtenidos variando los descriptores moleculares utilizados y teniendo en cuenta la significancia del coeficiente $\beta$ de cada variable. Fue seleccionado el modelo de relación entre tres variables desarrollado en la Ecuación 1, el cual explica solo un $68 \%$ de la varianza total de los valores 
experimentales de CC50

$$
\begin{array}{r}
\text { CC }_{50}=17,03-14,75 \text { C.F- 9,61 LUMO }-3,93 q 3 \\
R^{2}=0,68 ; \quad F=9,881 ; p=0,0009312
\end{array}
$$

En este modelo el coeficiente de la variable q3 no tiene un valor significativo debido a que el valor de $\mathrm{p}$ considerado para el coeficiente $\beta$ se encuentra por debajo del valor umbral de 0,05 fijado para este trabajo. Por lo tanto, se propone el modelo representado en la Ecuación 2 obtenido luego de la remoción del compuesto bis-THTT5, considerado un valor atípico teniendo en cuenta los resultados de la aplicación del test de Bonferronni (valor t-student $(-2,92051) \mathrm{p}$ no ajustado $(0,011929) \mathrm{p}$ de Bonferonni $(0,21472))$.

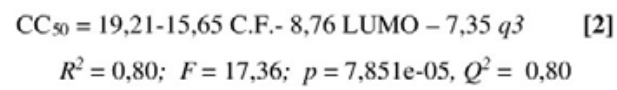

La variable con mayor grado de influencia en la respuesta es el C.F, el signo negativo indica que un cambio en la estructura molecular base que conlleve a un aumento del C.F. produce una disminución en la CC50, lo que se traduce en un compuesto más tóxico. El mismo efecto, aunque en menor medida, se lograría si el cambio estructural conllevara a un aumento de la energía del LUMO o la carga parcial en la posición tres del anillo. Removiendo bis-THTT5 el coeficiente $\beta$ asociado a q3 puede ser tenido en cuenta con un nivel se significación $\alpha$ menor que 0,05 . La capacidad predictiva del modelo fue evaluada internamente a través de estadístico Q2 evidenciándose la no existencia de sobre-ajuste a los datos de entrenamiento

Modelo para el análisis de la actividad: Para el modelo de asociado a la actividad, de igual manera fue ensayada una estrategia de selección que incluye realizar los modelos utilizando los valores experimentales de CI50 y su transformación logarítmica (PCI50). Desafortunadamente, ninguno de los dos caminos arrojó resultados satisfactorios para un ajuste lineal. Consideramos en este trabajo el modelo representado en la Ecuación 3 con resultados discretos teniendo en cuenta que se está trabajando con datos experimentales. De las variables tenidas en cuenta para el estudio solo el C.F tiene significación para $\alpha$ menor que 0,05 , el modelo logra explicar solo el $47 \%$ de la varianza total. La ecuación representada a continuación fue obtenida con la remoción del compuesto bis-THTT7, clasificado como valor atípico y posible valor influyente teniendo en cuenta el valor de $\mathrm{p}$ ajustado del test de Bonferronni ( $p=0,0022781)$. 


$$
\begin{aligned}
\mathrm{CI}_{50} & =9,61-5,42 \text { C.F } \\
R^{2} & =0,47 ; F=13,13 ; p=0,0025 \quad Q^{2}=0,23
\end{aligned}
$$

Debido a que el valor de Q2 evidencia un bajo poder predictivo, el modelo descrito no se propone para realizar análisis de predicción.

Relación actividad-citotoxicidad: Ambos modelos contienen como principal variable influyente al C.F, en igual sentido del efecto que produce. Un cambio en la estructura molecular base que conlleve a una disminución del C.F provocaría un aumento en el valor de CC50 del compuesto y el resultado sería un compuesto menos citotóxico. Sin embargo, el mismo efecto provocaría un compuesto menos activo.

Los descriptores q3 y LUMO podrían tenerse en cuenta a la hora de diseñar nuevas estructuras. Una disminución de la carga parcial en la posición tres del núcleo tiadiazínico favorece el aumento de la CC50, por lo que la presencia de grupos electro-donores en esta posición disminuirá la citotoxicidad de los compuestos; un mismo efecto provocaría una disminución en la energía del LUMO

Los compuestos bis-THTT5 y bis-THTT7, eliminados del análisis para la obtención de los modelos seleccionados, son valores extremos de las variables correspondientes en cada caso (CC50 y CI50, respectivamente) (Tabla 1). Ambos compuestos fueron incluidos en la muestra mediante el método de imputación por razones de tamaño de muestra, pero estos fueron reportados como valores en un intervalo.

Debido a las potencialidades de estos compuestos, se realizó un estudio de relación estructura-actividad a pesar de que pudiera considerarse limitado el número de derivados bis-THTT. Partiendo de este modelo podrán incluirse nuevos derivados en estudios posteriores.

\section{CONCLUSIONES}

Los modelos matemáticos obtenidos explican la manera en que se relacionan un conjunto de descriptores cuánticos y químico-físicos con la selectividad de derivados bis-THTT. El comportamiento mostrado por ambas variables en función del C.F es reflejo de la conducta de la mayoría los derivados tiadiazínicos recogidos en nuestra base de datos. Mantener esta variable constante y variar el resto de las variables significativas, podría constituir una estrategia en la obtención de nuevos derivados de esta familia. Para el análisis de ambas variables a la vez, la CI50 tendría una influencia considerablemente negativa en los resultados del modelo por lo que no debe utilizarse un Modelo General Lineal (MGL). 


\section{REFERENCIAS BIBLIOGRÁFICAS}

Alvar, J., Vélez, I. D., Bern, C., Herrero, M., Desjeux, P., Cano, J., Jannin, J., den Boer, M., WHO Leishmaniasis Control Team. (2012). Leishmaniasis Worldwide and Estimates of Incidence. PLoS ONE 7(5), e35671.

Coro, J., Atherton, R., Little, S., Wharton, H., Yardley, V., Alvarez, A., Súarez, M., Pérez, R., Rodríguez, H. (2006). Alkyl-linked bis-THTT derivates as potent in vitro tripanocidal agents. Bioorganic \& Medicinal Chemistry Letters 16(5), 1312-1315.

Coro, J., Little, S., Yardley, V., Suárez, M., Rodríguez, H., Martín, N., Pérez, R. (2008). Synthesis and Antiprotozoal Evaluation of New N4-(Benzyl) spermidyl-linked bis (1,3,5-thiadiazinane-2-thiones). Arch. Pharm. Chem. Life Sci. 341(11), 318-329.

Coro, J., Pérez, R., Rodríguez, H., Suárez, M., Vega, C., Rolón, M., Montero, D., Nogal, J. J., Gómez-Barrio, A. (2005). Synthesis and antiprotozoan evaluation of new alkyl-linked bis (2 -thioxo-[1,3,5]thiadiazinan-3yl-)carboxylic acids. Bioorg. Med. Chem. 13(10), 13: 3413-3421.

Coro, J., Piñeiro, R. P., Fidalgo, L. M., Cabrera, H. R., Navarro, M. S. (2011). Thiadiazine derivatives as antiprotozoal new drugs. Open Med Chem J 5:51-60.

Cramer, R. D., Bunce, J. D., Patterson, D. E., Frank, I. E. (1988). Cross validation, Bootstrapping, and Partial Least Squares Compared with Multiple Linear Regression in Conventional QSAR Studies. Quant. Struct.-Act. Relat. 18-25.

James, J. P. (2008). MOPAC2009, Stewart Computational Chemistry, Colorado Springs, CO, USA, HTTP://OpenMOPAC.net.

Lumley, T. (2001). Lumley Programmer's niche, Macros in R. R News 1:11-13. Monge-Maillo, B., López-Vélez, R. (2013). Therapeutic options for visceral leishmaniasis. Drugs 73(17): 1863-1888.

Monzote, L., Montalvo, A. M., Geigel, L. F., Pérez, R., Suárez, M., Rodríguez, H. (2004). Effect of Thiadiazine Derivatives on Intracellular Amastigotes of Leishmania amazonensis. Mem Inst Oswaldo Cruz 99(3), 329-330.

Nagle, A. S., Khare, S., Kumar, A.B., Supek, F., Buchynskyy, A., Mathison, C. J., Chennamaneni, N. K., Pendem, N., Buckner, F. S., Gelb, M. H., Molteni, V. (2014). Recent Developments in Drug Discovery for Leishmaniasis and Human African Trypanosomiasis. Chemical Reviews 114 (22), 11305-11347. 